\title{
Viel Gemüse und wenig Salz senken bei mir den Druck: Patientenbericht von Lilo Götte
}

Mein Blutdruck steigt unter anderem durch Stress. Mein Hausarzt hat bei einer Routineuntersuchung die erhöhten Werte festgestellt - zu meiner Überraschung. Ich war 58 Jahre alt und schlank, am Gewicht lag es daher wohl nicht. Und Zigaretten habe ich immer schon verschmäht. Als ich die Diagnose bekam, war das für mich ein Signal, mich intensiver um meine Gesundheit zu kümmern. Ich wollte mehr über die Ursachen für Bluthochdruck wissen und habe recherchiert. Dabei stieß ich darauf, dass ungesunde Ernährung ein Grund sein kann. Und ich beschloss - auch im Sinne meiner Familie -, in der Küche etwas zu ändern. Das Risiko, dass nicht nur ich, sondern auch die anderen eine Herzerkrankung oder einen Schlaganfall erleiden könnten, wollte ich auf jeden Fall vermeiden. Ich änderte meine Einkaufsliste und machte mich mit den typischen Rezepten der Mittelmeerküche wie einem französischen Ratatouille (Gemüsegericht mit Tomaten, Auberginen, Zucchini und Gurken) vertraut. Glücklicherweise kann ich gut kochen und spezielle Kochbücher haben die Umstellung noch gefördert. Außerdem schaue ich mir gern die „Ernährungsdocs“ an, die montags um 21 Uhr im 3. Programm des NDR „praktizieren“. Sie haben öfters Bluthochdruckpatienten mit erheblichem Übergewicht als Betroffene und verordnen ihnen viele Gemüsegerichte, viel Bewegung. Danach geht es ihnen besser.

Ich denke, Kochkurse helfen zusätzlich, die nötige Motivation zu fördern. Gerade Familien sollten darauf achten, dass die Kinder nicht übergewichtig werden. Wenn ich bedenke, wieviel Zucker schon die meisten Müslisorten enthalten! Es gibt Nährwerttabellen, die beim Einkauf unterstützen können. Wichtig für Hochdruckpatienten wäre es, gerade die Kombination von Kohlehydraten und Fetten in Form von Fertiggerichten, Fast Food, Pizza oder Pasta zu vermeiden. Sie treiben den Blutzuckerspiegel in die Höhe und können zu Übergewicht und dann zum Bluthochdruck führen. Auch zuckerhaltige Getränke gehören zu den Risikofaktoren, besser sind Mineralwasser oder Kräutertees, besonders Malventee.

Zur gesunden Ernährung gehört die Umstellung auf reichlich Gemüse, Obst und Fisch. Vor allem aber gilt es, Kochsalz sowie Zucker beim Zubereiten der Speisen einzuschränken, dafür mehr Kräuter und gesundes Öl zu verwenden. Wir beschlossen innerhalb der Familie, nur noch zwei Mal in der Woche Fleisch aufzutischen - und zwar pro Person nur etwa 300 Gramm. Grundsätzlich essen wir weniger Kohlenhydrate, also nur noch kleinere Mengen Brot oder Nudeln. Das

Ә OpenAccess. (c) 2021 Natascha Plankermann, publiziert von De Gruyter. (cc))BY-NC-SA Dieses Werk ist lizenziert unter einer Creative Commons Namensnennung - Nicht kommerziell - Weitergabe unter gleichen Bedingungen 4.0 International Lizenz. https://doi.org/10.1515/9783110713336-033 
Abendbrot haben wir auf etwa 18 Uhr vorverlegt, damit der Körper die Nahrung anschließend gut „verarbeiten“ kann.

Die Umstellung auf die gesunde Kost und mehr Bewegung ist gut gelungen. Außerdem nehme ich regelmäßig die verschriebenen Blutdruckmittel ein. Mein Blutdruck ist seit vielen Jahren normal und ich habe jetzt ein gutes Gewissen, mein Risiko gemindert zu haben. Wichtige Informationen dazu erhalte ich von der Deutschen Hochdruckliga. Zusammen mit meinem Arzt habe ich eine Selbsthilfegruppe gegründet, die seit vielen Jahren vom Göttinger Blutdruckinstitut und seinen Ärzten begleitet wird. Darüber sind wir sehr dankbar, denn wir möchten doch gerne gesund alt werden.

Lilo Götte (79) wohnt in Göttingen, von Beruf war sie Fremdsprachensekretärin. Zusammen mit ihrem Hausarzt hat sie vor Jahren bereits eine Selbsthilfegruppe gegründet, die inzwischen vom Göttinger Blutdruckinstitut unterstützt wird. Dadurch bekommen die Mitglieder viele wichtige Informationen aus erster Hand. Das Blutdruckinstitut Göttingen e. V. arbeitet in Kooperation mit dem Nephrologischen Zentrum Göttingen und dem Evangelischen Krankenhaus GöttingenWeende, mehr Informationen gibt es unter www.blutdruck-goe.de. 